\title{
Exploring the benefits of depositing hard TiN thin films by non-reactive magnetron sputtering
}

\author{
D. Martínez-Martínez** , C. López-Cartes, A. Fernández, J. C. Sánchez-López \\ Instituto de Ciencia de Materiales de Sevilla, Centro Mixto CSIC-Universidad de Sevilla, Avda. Américo \\ Vespucio 49, 41092-Sevilla, Spain \\ * Corresponding author: Tel. and fax: +32 (0)2 6502952. \\ E-mail address: $\underline{\text { d.martinez-martinez@m2i.nl }}$ \\ "Present address: Materials Innovation Institute, University of Groningen, Department of Applied \\ Physics, Nijemborgh 4, 9742 AG Groningen, The Netherlands.
}

\begin{abstract}
The aim of this paper is to compare the mechanical and tribological properties of TiN coatings prepared in a conventional magnetron sputtering chamber according to two different routes: the usual reactive sputtering of a Ti target in an $\operatorname{Ar} / \mathrm{N}_{2}$ atmosphere vs. the comparatively more simple sputtering of a TiN target in a pure Ar atmosphere. Improved properties in term of hardness and wear rates were obtained for films prepared by non-reactive sputtering route, due to the lower presence of oxynitride species and larger crystalline domain size. Additionally, a significant hardness enhancement (up to $45 \mathrm{GPa}$ ) is obtained when a $-100 \mathrm{~V}$ d.c. bias is applied during growth. This behaviour is explained by non-columnar growth and small grain size induced by effective ion bombarding. These results demonstrate that non-reactive sputtering of TiN target appears a simple and efficient method to prepare hard wearresistant TiN films.
\end{abstract}

Keywords: TiN, target, magnetron sputtering, XRD, stress, hardness 


\section{Introduction}

TiN-based coatings have been largely the most studied material for protective purposes, due to their optimal mechanical properties (high hardness and elastic modulus) and good wear and corrosion resistance [1-6]. Besides, the gold colour of TiN when prepared in stoichiometric state qualifies it for decorative purposes. In particular, great attention has been paid in the literature to the incorporation of additional elements or phases in order to improve its performance. For example, the oxidation resistance can be increased by including metals as $\mathrm{Al}, \mathrm{Si}, \mathrm{B}, \mathrm{Cr} . .[7-10]$; its friction coefficient can be reduced by the formation of (nano)composites with lubricant phases as amorphous carbon [11-13] or $\mathrm{MoS}_{2}$ [14]; and the wear resistance can be enhanced by the formation of a $\mathrm{TiN} / \mathrm{a}-\mathrm{Si}_{3} \mathrm{~N}_{4}$ nanocomposite [15].

Among many deposition techniques employed to deposit this material, physical vapour deposition methods are the most used, including pulsed laser deposition [16], electron beam evaporation [17], cathodic arc ion-plating [18], or magnetron sputtering [19-20], being this latter technique possibly the most common. Vast majority of the published works in the literature use a reactive route for its preparation with the $\mathrm{Ti}$ target working in fully poisoned regime (referred to as compound sputtering mode) or in the transition mode. Growth of stoichiometric compound films with relatively high rates can be facilitated in the transition regime between the metallic and the compound mode [21]. However, the relationship between reactive gas flow and process parameters is complex leading to an unstable transition zone and a hysteresis in the process parameters, Thus, the use of feedback control devices becomes necessary in order to control the degree of poisoning of the target during deposition. In addition, nitrogen is a very stable molecule, and much energy is needed to break its three-fold bond, particularly in the excitation of its vibrational modes [22]. Only in few cases the 
deposition has been performed by the comparatively simple sputtering from a TiN target [23-25], and its potential has not been deeply explored yet. The aim of this paper is to compare both synthetic routes for preparation of $\mathrm{TiN}$ in the same conventional magnetron sputtering chamber: the usual one (i.e. reactive sputtering of $\mathrm{Ti}$ in an $\mathrm{Ar} / \mathrm{N}_{2}$ atmosphere) and non-reactive sputtering of TiN in a pure Ar atmosphere. The effect of the application of a d.c. negative bias on the substrates during growth is also studied.

\section{Experimental details}

\subsection{Sample preparation}

TiN coatings were prepared on AISI M2 polished steel $(\mathrm{H}=16 \mathrm{GPa}), \mathrm{NaCl}$, and $\mathrm{Si}$ (100) substrates by r.f. sputtering of titanium nitride (Goodfellow, 99\% purity) or titanium (Goodfellow, 99.99\% purity) targets in $\mathrm{Ar}$ or $\mathrm{Ar} / \mathrm{N}_{2}$ discharges (2 $\mathrm{sccm} \mathrm{N}, 7 \%$ in volumetric flow), respectively. The applied power was $250 \mathrm{~W}$ and the target diameter 2 inches. No intentional heating was applied to the substrates. The base pressure of the vacuum chamber was found to be lower than $6.5 \times 10^{-4} \mathrm{~Pa}$ and about $0.75 \mathrm{~Pa}$ during deposition. Unbiased and d.c. negative biased $(-100 \mathrm{~V})$ films were prepared in both cases. A prior underlayer (ca. $250 \mathrm{~nm}$ thick) of Ti or TiN was deposited for adhesion improvement using the same sputtering power and $100 \mathrm{~V}$ of negative bias. More details concerning film preparation can be found elsewhere [11-12]. The specific synthesis conditions and properties of the four films under study are summarized in Table 1 . The total film thickness varied in the range of 300 to $800 \mathrm{~nm}$, with larger deposition rates for samples prepared by non-reactive sputtering, in agreement with what reported previously by Mayrhofer et al. [23]. Coatings were labeled according to the nitrogen source (gas or target); i.e. "G" if prepared by sputtering of a Ti target in a $\mathrm{Ar} / \mathrm{N}_{2}$

atmosphere (reactive) or " $\mathrm{T}$ " if prepared by sputtering of a TiN target in a Ar 
atmosphere (non-reactive). The application of the bias to the substrates is indicated with a small ' $b$ ' as suffix.

\subsection{Sample characterization}

Grazing angle X-ray diffraction (GAXRD) measurements were carried out using $\mathrm{Cu} \mathrm{K} \mathrm{K}_{\alpha}$ radiation in a Siemens D5000 diffractometer at an incidence angle of $1^{\mathrm{o}}$. The macrostrain $\left(\varepsilon_{M}\right)$ was estimated from the position of the (220) peak through the following expression:

$$
\varepsilon_{M}=\frac{d-d_{0}}{d_{0}}=\frac{\frac{\lambda}{2 \sin \theta}-d_{0}}{d_{0}}
$$

Where $\lambda$ is the wavelength of the used X-rays, $\theta$ is the Bragg's angle and $d$ and $d_{0}$ are the (220) interplanar distances measured on the sample and an unstressed reference, respectively. To estimate the microstrain $\left(\varepsilon_{m}\right)$ of the coatings, the Williamson-Hall's method has been employed [26-27]. According to this approach, the broadening $(\beta)$ of a XRD peak with a Lorentzian shape can be written as the sum of the instrumental broadening $\left(\beta_{i}\right)$, plus the broadening related to the crystal size $\left(\beta_{C S}\right)$, and the broadening due to microstrain $\left(\beta_{M S}\right)$ :

$$
\beta=\beta_{i}+\beta_{C S}+\beta_{M S}=\beta_{i}+\frac{0,9 \lambda}{t \cos \theta}+4 \varepsilon \operatorname{tag} \theta
$$

Where $t$ is the crystal size. The microstrain was obtained from the slope of the curve by plotting $\left(\beta-\beta_{i}\right) \cos \theta / \lambda$ versus $\sin \theta / \lambda$. In general, the higher the slope, the higher the microstrain. The Scherrer's equation was preferred to estimate the grain size from the broadening of the (220) peak due to the high uncertainty in the determination of the ordinate at the origin in the Williamson-Hall method [28]. 
Scanning electron microscopy (SEM) analysis was done in a Hitachi S5200 field emission microscope operating at $5 \mathrm{kV}$. X-ray photoelectron spectroscopy (XPS) was performed in a VG-Escalab 210 spectrometer working in the constant analyzer energy mode with a pass-energy of $50 \mathrm{eV}$ and non-monochromatic $\mathrm{Mg} \mathrm{K}_{\alpha}$ radiation (1253.6 $\mathrm{eV}$ ) as excitation source. The chemical analysis corresponds to the sample surface in the as-deposited state after exposure to air. No argon ion etching was used prior to analysis to avoid chemical bonding modifications.

The tribological properties of the coatings were evaluated by ball-on-disk friction tests in unlubricated sliding against AISI52100 6-mm steel balls in ambient air at relative humidity between $40-50 \%$. The test parameters were set to $5 \mathrm{~N}$ of applied load (maximum initial Hertzian contact pressure $1.12 \mathrm{GPa}$ ), $10 \mathrm{~cm} / \mathrm{s}$ of linear speed, and $1000 \mathrm{~m}$ of sliding distance. Normalized wear rates $\left(\mathrm{mm}^{3} / \mathrm{N} \cdot \mathrm{m}\right)$ were evaluated from cross-sectional profiles taken across the disk wear track after testing by means of stylus profilometry.

The microhardness, reduced Young's modulus, and elastic work were measured on coated M2 steel disks with a Fischerscope H100 dynamic microprobe instrument using a conventional Vickers indenter at loads up to $10 \mathrm{mN}$. The maximum load was selected in such a way that the maximum indentation depth did not exceed the $10-15 \%$ of the coating thickness in order to avoid the influence of the substrate. The test parameters were fixed to an initial load of $0.4 \mathrm{mN}$ up to a maximum load of $10 \mathrm{mN}$ in 40 load steps, with a time between two load steps of 0.5 seconds and the test cycle was loading and unloading. All the parameters were calculated from the load-unload displacement curves by the Fischerscope software. 
To calculate the values of film residual stress, the Stoney's equation has been employed. The curvature was estimated by measuring $20-\mathrm{mm}$ profiles on coatings deposited over $525 \mu \mathrm{m}$ thick silicon (100) substrates.

\section{Results and discussion}

Figure 1 shows the GAXRD diffraction patterns of the four samples in the $2 \theta$ range $\left(50-70^{\circ}\right)$ around the position of the $\mathrm{TiN}$ (220) peak. This region has been selected to appreciate better the influence of structural effects. On the one hand, the (220) peak is situated at a relatively high angle, where peak displacements can be more easily observed, and exhibits a good intensity. On the other hand, the reflections due to the Ti underlayer and Fe from the substrate can be discriminated from the (220) TiN peak. The first evidence is the good agreement of the peak positions with the reference values of a cubic TiN phase (JCPDS card 38-1420) for the samples prepared without bias ( $\mathrm{G}$ and $\mathrm{T}$ samples). However, the analogous samples prepared with bias show a displacement to lower angles as a result of a Possion expansion of the lattice in the direction perpendicular to the substrate caused by compressive stress. Based on the (220) peak position, the macrostrain of the plane has been calculated (see Table 1). Both unbiased samples show low values of macrostrain, while the application of the bias leads to a lattice expansion. This effect is more obvious for samples prepared from a TiN target ( $\varepsilon_{M}$ changes from $-0.1 \%$ to $0.7 \%$ ). In both routes, important peak broadening is noted when bias is used, which indicates a diminution of the grain size and an increment of the alterations of the lattice induced by micro-strain. This is caused by an increment of the nucleation points and the interruption of the grain growth by increased flux of impinging ions, in good agreement with previous works [23]. To evaluate the contribution of the microstrain in the TiN grains, Williamson-Hall plots have been used (see Figure 2). The slopes of the lines are not very high, indicating low values of 
microstrain, although those corresponding to the biased samples are always higher, $\left(\varepsilon_{m}\right.$ $\sim 0.5 \%)$ versus $\left(\varepsilon_{m} \sim 0.1-0.3 \%\right)$ for the unbiased ones. As the values of the y-axis are inversely proportional to the grain size (see Experimental details), the higher values obtained for the biased samples are indicative of smaller grain sizes. This trend agrees with that obtained by using the Scherrer's equation, as can be concluded from the values summarized in Table 1. It can be seen that both samples prepared with bias showed lower average grain sizes $(8-10 \mathrm{~nm})$ than the ones prepared without bias $(>15 \mathrm{~nm})$. It is noted that samples prepared by reactive sputtering of Ti target show smaller grain sizes. This is caused by a lower energy transfer to the $\mathrm{N}$ atoms (and therefore a more restricted mobility of the adatoms on the surface), since an important contribution is used in exciting vibrational modes of the $\mathrm{N}_{2}$ molecule [23]. A similar effect is reported by Kawamura et al. [25], who observed a grain size reduction when introducing $\mathrm{N}_{2}$ during direct TiN sputtering.

Figure 3 shows the deconvolution of the Ti $2 p$ XPS peak of one sample for each series. The peak appears as a doublet due to the spin-orbital interaction. Three chemical environments can be distinguished for the Ti $2 \mathrm{p}_{3 / 2}$ component at $458.5 \mathrm{eV}$ (Ti-O [2930]), $456.8 \mathrm{eV}$ (attributable to an oxynitride peak, TiON [31], and/or a shake-up satellite [32-33]), and $455.3 \mathrm{eV}$ (Ti-N [34-35]). It can be seen that the intensity of the Ti-N component is higher than TiON in the non-reactive sample (area ratio of 1.2), while in the reactive one the opposite is seen (area ratio of 0.6 ). The same behaviour has been observed in TiN/a-C nanocomposite films using both synthetic routes (reactive and nonreactive) including a carbon target [12]. This indicates that, under our experimental conditions, the contribution of the TiN phase becomes more important when sputtering directly a TiN target, in good agreement with the XRD data. This issue is of particular 
importance in case of industrial chambers, where the presence of oxygen contaminations cannot be reduced as in the case of a lab-scale equipment.

To summarize the conclusions of the structural and chemical bonding investigations carried out on both series, we can state that the bias reduces the crystalline grain size and causes lattice deformation, denoted as micro and macro-strain effects; and the choice of a non-reactive sputtering leads to larger grain sizes and lower oxygen incorporation into the TiN film.

The mechanical and tribological properties of the samples under study are summarized in Table 1. It can be seen that the friction coefficients are rather similar, around 0.6-0.7, as expected for these hard films under these tribological conditions [36]. Regarding the normalized wear rates, higher resistance is obtained for the $\mathrm{T}$ samples, prepared by direct sputtering of the TiN target. It must be noted that the high wear obtained for the G sample is due to poor adhesion. The worst mechanical properties are seen for the sample prepared in $\mathrm{N}_{2}$ atmosphere in absence of bias. Hardness and reduced Young's modulus can be increased by the application of a substrate bias or using a TiN target. This latter factor appears dominant since the T sample (without bias) shows even better values than the $\mathrm{Gb}$, with bias assistance. This is probably related to the increased presence of TiON species on the samples prepared using $\mathrm{N}_{2}$. An outstanding hardness enhancement (up to $45 \mathrm{GPa}$ ) is noticed for the sample $\mathrm{T}$ when the bias is applied. Similar values have been obtained by other authors by using optimised values of ion energy and flux [23]. Shin et al. [37] reported a significant hardness increase of $12 \mathrm{GPa}$ for $\operatorname{TiN}_{\mathrm{x}}$ layers when $x$ changed from 1 to 0.67 . In the present case, the $\mathrm{Ti} / \mathrm{N}$ ratio measured by electron energy-loss spectroscopy [12] is constant and near to 1:1 stoichiometry in all cases (cf Table 1). Moreover, this sample shows also an outstanding value of elastic work of $92.4 \%$, almost double of any of the others. 
The film in-plane residual stress $(\sigma)$ is always found to be compressive type, being relatively low $(<1 \mathrm{GPa})$ for both samples prepared without bias, but increasing significantly when bias is applied (larger than $6 \mathrm{GPa}$ ). This observation agrees with the displacement of the XRD peaks to lower angles and the higher values of the macrostrain observed when using bias. However, the origin of the XRD peak displacements can be also due to lattice imperfections, like site vacancies, substitution and displacement of atoms on interstitial lattice sites (Schotty and Frenkel defects [23]) or presence of heteroatoms (Ar, O). In order to discriminate between both possibilities, an extended grazing XRD measurement at higher angles has been carried out (see Figure 4) on these same samples. The diffraction peaks from the sample grown with bias are broader due to its lower grain size. Furthermore, it can be seen that the peak displacement due to the bias application is different depending on the measured range: while at low angles the peak shifted towards lower angles, at high angles $\left(>100^{\circ}\right)$ the displacement occurs in the opposite direction.

This effect can be explained taking into account the particular geometry of GIXRD measurements. In typical Bragg-Brentano configuration, only the planes parallel to the surface are detected. Thus, when measuring a film with residual compressive stress what is indeed measured corresponds to a Poisson's expansion of the lattice. Under this configuration, a compressive stress is always manifested as a displacement of the diffraction peaks to lower angles independently on the considered Bragg's reflection. Otherwise, in a grazing angle configuration, the angle between the surface and the radiation source is fixed (and low), so the angle between the detected planes and the surface increases with the $\theta$ angle. Thus, for low values of $\theta$, the detected planes are more parallel to the surface, and therefore under a higher influence of the Poisson's expansion than the compression (see scheme in Figure 4). When $\theta$ increases, 
the detected planes are more perpendicular to the surface, so they become more and more affected by the compression forces. Thus, at high angles, we are detecting planes which are more influenced by the compression than by the Poisson's expansion, and as a result, the peak shifts to higher angles. By assembling all these findings we can thus conclude that the origin of the peak displacement is mainly attributed to film compressive stress, since the deformation of the lattice due to contaminants, point defects and/or vacancies would cause the same direction of displacement in all the diffractogram, even in grazing angle configuration.

The growth of the samples prepared by the non-reactive route was further investigated by cross-sectional SEM analysis in order to understand the observed hardness enhancement. Figure 5 shows SEM images of the T samples both in top and cross-section views. Fig. 5a corresponds to the sample prepared without bias (top view) revealing triangular equiaxed grains, indicative of a faceted $<111>$ columnar growth, as confirmed by the analysis of the cross-section morphology (Fig. 5b). The sample prepared with bias assistance (cf. Fig. 5c and 5d) shows a more compact structure where the columns cannot be clearly appreciated. The open columnar structure agrees with the higher values of grain size observed in $\mathrm{T}$ samples, whilst the finer structure of $\mathrm{Tb}$ is in concordance with the diminution of the grain sizes. The densification of the structure and the presence of stresses by the influence of bias agree with the model of Chanson et al. [38], where the high mobile adatoms diffuse through the grain boundaries leading to high stress and compactness. High substrate biases are known to lead to high in-plane compressive stress, and large residual defect density [39-41], which are in agreement with the larger microstrains observed when biasing (cf. Table 1). In contrast, less compact intercolumnar boundaries allow the generated stresses to be relaxed at open voided boundaries. 
Similarly high compressive stress values have been also previously reported in case of thinner films deposited by reactive route [42-43], which are observed to decrease with the thickness. This behavior has been reproduced here, with the deposition of an unbiased film on top of a biased one (sample $\mathrm{Tb}$ is the underlayer used in sample T). The existence of stress gradient in sputtered TiN films has been explained in terms of the of the initial growth in the $<001>$ direction, followed by $<111>$ oriented crystals as the film grows thicker [44]. The former orientation is a more open channeling direction for impinging particles from ion bombardment than the latter one, which would explain the high stresses observed at the beginning of the growth. Another explanation for the stress gradient is based in an initial compressive stress due to the growth of induced point-defects, followed by a tensile stress behavior due to intercolumnar attractive forces [45]. Both explanations agree with the observed stress reduction after deposition of a $<111>$ columnar film on top of the biased dense one.

The decrease of average grain sizes, the high density of defects and grain boundaries, the existence of micro and macrostrain correlate well with the observed enhancement of hardness as they diminish the dislocation mobility. Nevertheless, there are also additional factors influencing the mechanical properties, since both samples prepared with bias ( $\mathrm{Tb}$ and $\mathrm{Gb}$ ), which show comparable stress and crystallite sizes, exhibit very different hardness values and elastic recovery. This difference probably arises from presence of oxynitride species, as commented before, originated when using reactive sputtering. In fact, the stoichiometry of TiN samples is known to influence the mechanical properties of TiN films [37]. In addition, the overall amount of energy supplied to the growing film and the energy per incident particle influence film growth $[23,43]$, which is favored in the absence of nitrogen in the gas phase. 


\section{Conclusions}

Four TiN films have been prepared by two different routes by magnetron sputtering, reactive and non-reactive, and with or without bias assistance. The samples prepared by reactive sputtering of a Ti target using a $\mathrm{Ar} / \mathrm{N}_{2}$ mixture show smaller grain size and more TiON species than their corresponding ones prepared by direct sputtering of a TiN target with Ar. Additionally, they also show also worse wear resistance and lower mechanical properties. The bias reduces the grain size and causes lattice distortion exhibiting micro- and macro-strains. The mechanical properties are improved, especially for the samples prepared from a TiN target (hardness up to $45 \mathrm{GPa}$ and elastic work $>90 \%$ ). This improvement is mainly caused by the replacement of the columnar structure by a dense morphology due to the application of a bias, besides of the low grain size, lattice distortion and reduced presence of oxynitride species. Therefore, the non-reactive route appears as a simple option to prepare TiN thin films with good mechanical and tribological performance, which results of practical interest for many applications in which this material is used for protective and decorative purposes.

\section{Acknowledgments}

The authors are grateful to the Spanish Ministry of Economy and Competitiveness (projects N ${ }^{\circ}$ MAT2010-21597-C02-01, MAT2011-29074-C02-01 and Consolider FUNCOAT CSD2008-00023) and Junta de Andalucía (P10-TEP 06782). M. 
Brizuela and A. Garcia-Luis from INASMET-Tecnalia are acknowledged for the hardness measurements. 


\section{References}

[1] J.E. Sundgren, B.O. Johansson, S.E. Karlsson, Thin Solid Films 105 (1983) 353366.

[2] J.E. Sundgren, B.O. Johansson, S.E. Karlsson, H.T.G. Hentzell, Thin Solid Films 105 (1983) 367-384.

[3] G. Lemperiere, J.M. Poitevin, Thin Solid Films 111 (1984) 339-349.

[4] U. Helmersson, B.O. Johansson, J.E. Sundgren, H.T.G. Hentzell, P. Billgren, J. Vac. Sci. Technol. A-Vac. Surf. Films 3 (1985) 308-315.

[5] J. Pelleg, L.Z. Zevin, S. Lungo, N. Croitoru, Thin Solid Films 197 (1991) 117128.

[6] P. Patsalas, C. Charitidis, S. Logothetidis, Surf. Coat. Technol. 125 (2000) 335340.

[7] S. PalDey, S.C. Deevi, Mater. Sci. Eng. A 342 (2003) 58-79.

[8] P. Steyer, D. Pilloud, J.F. Pierson, J.P. Millet, M. Charnay, B. Stauder, P. Jacquot, Surf. Coat. Technol. 201 (2006) 4158-4162.

[9] M. Pfeiler, J. Zechner, M. Penoy, M. Michote, C. Mitterer, M. Kathrein, Surf. Coat. Technol. 203 (2009) 3104-3110.

[10] G.S. Fox-Rabinovich, S.I. Kovalev, M.H. Aguirre, B.D. Beake, K. Yamamoto, S.C. Veldhuis, J.L. Endrino, D.L. Wainstein, A.Y. Rashkovskiy, Surf. Coat Technol. 204 (2009) 489-496. 
[11] D. Martinez-Martinez, C. Lopez-Cartes, A. Fernandez, J.C. Sanchez-Lopez, Surf. Coat. Technol. 203 (2008) 756-760.

[12] D. Martinez-Martinez, C. Lopez-Cartes, A. Fernandez, J.C. Sanchez-Lopez, Solid State Sci. 11 (2009) 660-670.

[13] H.C. Barshilia, M.S. Prakash, D.V. Sridhara Rao, K.S. Rajam, Surf. Coat. Technol. 195 (2005) 147-153.

[14] R. Gilmore, M.A. Baker, P.N. Gibson, W. Gissler, M. Stoiber, P. Losbichler, C. Mitterer, Surf. Coat. Technol. 108 (1998) 345-351.

[15] M. Diserens, J. Patscheider, F. Levy, Surf. Coat. Technol. 109 (1998) 241-246.

[16] J. Narayan, P. Tiwari, X. Chen, J. Singh, R. Chowdhury, T. Zheleva, Appl. Phys. Lett. 61 (1992) 1290-1292.

[17] L.I. Wei, C. Jun-Fang, Appl. Surf. Sci. 253 (2007) 7019-7023.

[18] T. Ikeda, H. Satoh, Thin Solid Films 195 (1991) 99-110.

[19] F. Elstner, A. Ehrlich, H. Giegengack, H. Kupfer, F. Richter, J. Vac. Sci. Technol. A 12 (1994) 476-483.

[20] M. Kawamura, Y. Abe, H. Yanagisawa, K. Sasaki, Thin Solid Films 287 (1996) 115-119.

[21] S. Berg, T. Nyberg, Thin Solid Films 476 (2005) 215-230.

[22] F. Debal, J. Bretagne, M. Jumet, M. Wautelet, J.P. Dauchot, M. Hecq, Plasma Sources Sci. Technol. 7 (1998) 219-229. 
[23] P.H. Mayrhofer, F. Kunc, J. Musil, C. Mitterer, Thin Solid Films 415 (2002) 151-159.

[24] C. Mitterer, P.H. Mayrhofer, W. Waldhauser, E. Kelesoglu, P. Losbichler, Surf. Coat. Technol. 108-109 (1998) 230-235.

[25] M. Kawamura, K. Kumagai, Y. Abe, K. Sasaki, H. Yanagisawa, Vacuum 51 (1998) 377-380.

[26] G.K. Williamson, W.H. Hall, Acta Metall. 1 (1953) 22-31.

[27] B.D. Cullity, S.R. Stock, Elements of X-Ray Diffraction, Prentice Hall, New Jersey, 2001.

[28] C. Mansilla, Solid State Sci. 11 (2009) 1456-1464.

[29] D. Leinen, A. Fernandez, J.P. Espinos, J.P. Holgado, A.R. Gonzalez-Elipe, Appl. Surf. Sci. 68 (1993) 453-459.

[30] D. Briggs, M.P. Seah, Practical surface analysis, Willey \& Sons, 1993.

[31] P. Prieto, R.E. Kirby, J. Vac. Sci. Technol. A 13 (1995) 2819-2826.

[32] S. Lee, O. El-bjeirami, S.S. Perry, S.V. Didziulis, P. Frantz, G. Radhakrishnan, J. Vac. Sci. Technol. B 18 (2000) 69-75.

[33] S.V. Didziulis, J.R. Lince, T.B. Stewart, E.A. Eklund, Inorg. Chem. 33 (1994) 1979-1991.

[34] I. Bertoti, Surf. Coat. Technol. 151 (2002) 194-203.

[35] E.C. Onyiriuka, Appl. Spectrosc. 47 (1993) 35-37. 
[36] I.L. Singer, S. Fayeulle, P.D. Ehni, Wear 149 (1991) 375-394.

[37] C.S. Shin, D. Gall, N. Hellgren, J. Patscheider, I. Petrov, J.E. Greene, J. Appl. Phys. 93 (2003) 6025-6028.

[38] E. Chason, B.W. Sheldon, L.B. Freund, J.A. Floro, S.J. Hearne, Phys. Rev. Lett. 88 (2002) 156103.

[39] G. Abadias, Y.Y. Tse, P. Guerin, V. Pelosin, J. Appl. Phys. 99 (2006) 113519.

[40] I. Petrov, P.B. Barna, L. Hultman, J.E. Greene, J. Vac. Sci. Technol. A 21 (2003) S117-S128.

[41] L. Hultman, J.E. Sundgren, J.E. Greene, D.B. Bergstrom, I. Petrov, J. Appl. Phys. 78 (1995) 5395-5403.

[42] R. Machunze, G. Janssen, Surf. Coat. Technol. 203 (2008) 550-553.

[43] R. Machunze, F.D. Tichelaar, G. Janssen, Surf. Coat. Technol. 205 (2010) 13131319.

[44] R. Machunze, G. Janssen, Thin Solid Films 517 (2009) 5888-5893.

[45] G. Abadias, P. Guerin, Appl. Phys. Lett. 93 (2008) 111908. 


\section{Figure captions}

Figure 1. (color online) XRD patterns of the TiN coatings on the $50-70^{\circ}$ range. Left: samples prepared using reactive sputtering with $\mathrm{N}_{2}$. Right: samples prepared by nonreactive sputtering.

Figure 2. (color online) Williamson-Hall analysis of the XRD patterns. Top: TiN coatings prepared by reactive sputtering. Bottom: samples prepared by non-reactive sputtering.

Figure 3. (color online) Deconvolution of the Ti $2 \mathrm{p}$ high resolution XPS spectra. Left: sample prepared using reactive sputtering. Right: sample prepared by non-reactive sputtering.

Figure 4. (color online) Bias effect on the extended GAXRD diffractograms of the samples prepared by sputtering of a TiN target. The scheme represents the effect of compressive stress and Poisson`s expansion on the planes under analysis at low and high Bragg angles. See text for details.

Figure 5. SEM images of the samples prepared by non-reactive sputtering of a TiN target. Top: non-biased sample. Bottom: biased sample. Left: top views. Right: crosssections. All the scale bars represent $500 \mathrm{~nm}$. 
Table 1. Synthesis conditions and properties of the TiN films.

\begin{tabular}{|c|c|c|c|c|c|c|c|c|c|c|c|c|c|c|}
\hline \multirow{2}{*}{ Sample } & \multicolumn{3}{|c|}{ Synthesis conditions } & \multirow{2}{*}{$\begin{array}{c}\text { Deposition } \\
\text { rate } \\
(\mathrm{nm} / \mathrm{min})\end{array}$} & \multirow{2}{*}{$\begin{array}{l}\mathrm{Ti} / \mathrm{N} \\
\text { ratio }\end{array}$} & \multicolumn{3}{|c|}{ XRD data } & \multicolumn{6}{|c|}{ Tribo-mechanical properties } \\
\hline & Target & Atmosphere & $\begin{array}{c}\text { Bias } \\
(\mathrm{V})\end{array}$ & & & $\begin{array}{c}\text { Grain } \\
\text { size }(\mathrm{nm})\end{array}$ & \begin{tabular}{|c|} 
Microstrain \\
$(\%)$
\end{tabular} & $\begin{array}{c}\text { Macrostrain } \\
(\%)\end{array}$ & $\mathrm{f}$ & $\begin{array}{c}\mathrm{k} \\
\left(\times 10^{6} \mathrm{~mm}^{3} \mathrm{~N}^{-1} \mathrm{~m}^{-1}\right) \\
\end{array}$ & $\begin{array}{c}\mathrm{H} \\
(\mathrm{GPa}) \\
\end{array}$ & $\begin{array}{c}\mathrm{E}^{*} \\
(\mathrm{GPa})\end{array}$ & \begin{tabular}{|l|}
$\mathrm{We}$ \\
$(\%)$ \\
\end{tabular} & $\begin{array}{c}\sigma \\
(\mathrm{GPa})\end{array}$ \\
\hline G & $\mathrm{Ti}$ & $\mathrm{Ar} / \mathrm{N}_{2}$ & 0 & 2.5 & 1.1 & 18 & 0.3 & -0.3 & 0.65 & failure & 11.4 & 164 & 37.4 & 0 \\
\hline $\mathrm{Gb}$ & $\mathrm{Ti}$ & $\mathrm{Ar} / \mathrm{N}_{2}$ & -100 & 1.0 & 1.0 & 8 & 0.5 & 0.4 & 0.66 & 13.1 & 14.7 & 179 & 50.7 & -8.1 \\
\hline $\mathrm{T}$ & $\mathrm{TiN}$ & Ar & 0 & 4.2 & 1.1 & 39 & 0.1 & -0.1 & 0.61 & 2.6 & 19.0 & 218 & 50.8 & -0.6 \\
\hline $\mathrm{Tb}$ & TiN & Ar & -100 & 1.8 & 1.1 & 10 & 0.5 & 0.7 & 0.74 & 4.9 & 44.8 & error* & 92.4 & -6.6 \\
\hline
\end{tabular}


$2 \theta\left({ }^{\circ}\right)$
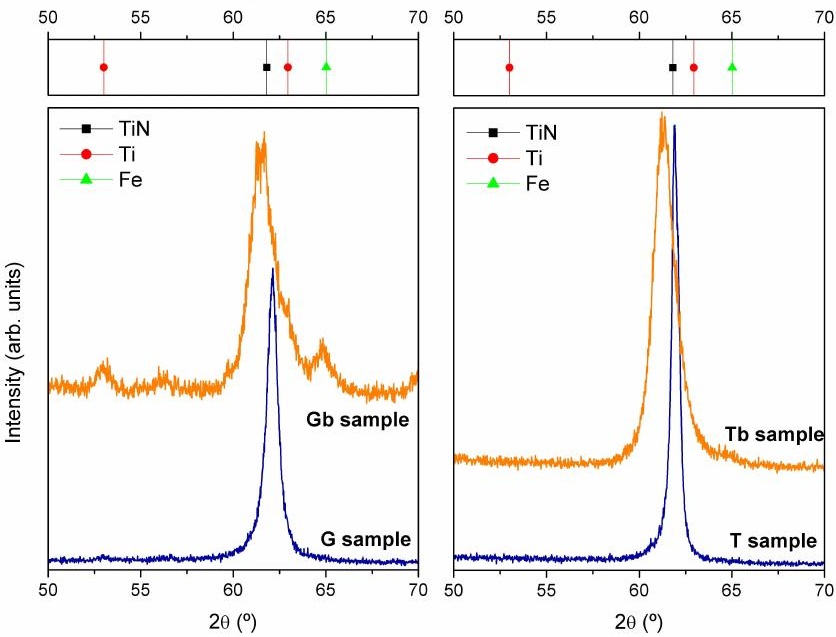
$\sin (\theta) / \lambda$

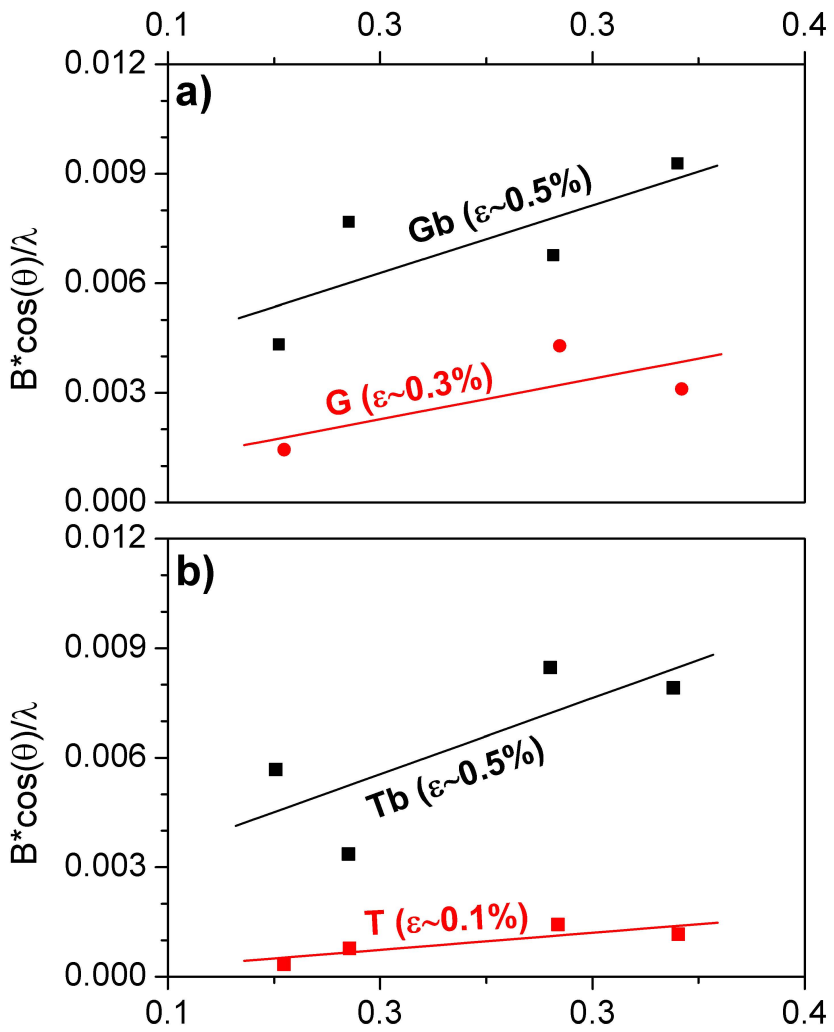

$\sin (\theta) / \lambda$ 


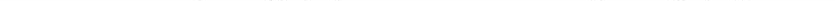


Poisson expansion
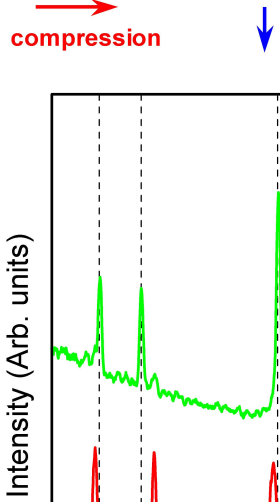

compression

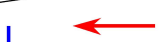

Poisson expansion $\uparrow$
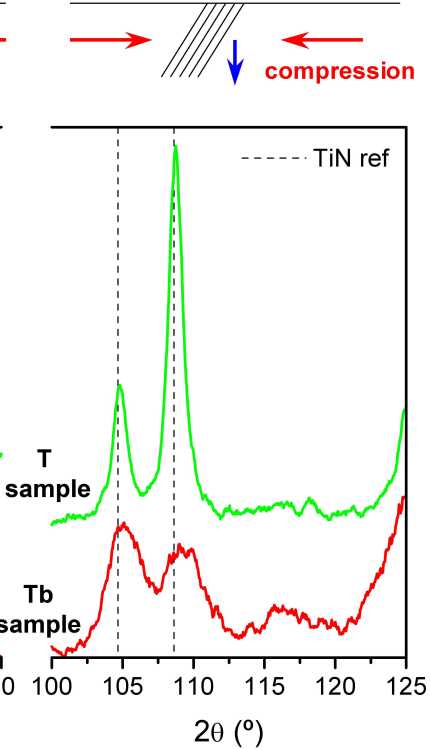
